Urologe 2013 · 52:931-932

DOI 10.1007/s00120-013-3230-5

Online publiziert: 27. Juni 2013

(c) Springer-Verlag Berlin Heidelberg 2013

R. Knüchel-Clarke ${ }^{1}$ A. Hartmann ${ }^{2} \cdot$ C. Stief ${ }^{3}$

${ }^{1}$ Institut für Pathologie, Uniklinik RWTH Aachen

${ }^{2}$ Institut für Pathologie, Uniklinikum Erlangen

${ }^{3}$ Klinik und Poliklinik für Urologie, Klinikum Großhadern, LMU München

\title{
Uropathologie für Urologen
}

Uropathologie für Urologen - warum sollte ich das lesen und „muss das wirklich sein"? Schauen Sie in die Beiträge dieses Heftes und überzeugen sie sich selbst! Mit der Abhandlung der häufigsten urologischen Tumoren (Prostata, Harnblase und Niere) möchten wir einen Beitrag zur interdisziplinären Kommunikation leisten, der der besseren Patientenversorgung dienen soll.

In den meisten Bereichen der Urologie ist die konventionelle histomorphologische Diagnostik nach makroskopischer Inspektion von Biopsiematerial und chirurgischen Resektaten immer noch der Goldstandard der Diagnostik und der wichtige Eckpfeiler für therapeutische Entscheidungen. Die wichtigsten Eckdaten der aktuellen Einteilung von Tumorgrad- und Tumorstadieneinteilung sind im ersten Teil des Heftes für Sie von erfahrenen Uropathologen zusammengefasst und in der Bedeutung akzentuiert dargestellt und anschaulich gemacht. Die Histologie wird ergänzt durch eine $\mathrm{Zu}$ sammenstellung der ergänzenden zytologischen Diagnostik, deren Stellenwert von morphologisch geschulten Urologen dargestellt wird.

Die standardisierte, leitliniengerechte und z. T. aufwändige histomorphologische Befundung ist eine elementare Grundlage der Prozessqualität der Pathologie, deren Wertigkeit ständig durch eine Korrelation mit den klinischen Daten prospektiv beurteilt werden sollte. Die Vorstellung von histologischen Diagnosen in der interdisziplinären Fallkonferenz parallel zu bildgebenden und klinischen Daten schafft gegenseitiges Verständnis für die Möglichkeiten und Grenzen der
Aussagen der diagnostischen Fächer und lehrt den Pathologen, in seiner Diagnostik auf die klinischen Entscheidungsprozesse einzugehen.

\section{》) Der interdisziplinäre \\ Austausch orientiert sich an bestehenden Leitlinien}

Der interdisziplinäre Austausch orientiert an bestehenden Leitlinien, ist eine zweite wesentliche Maßnahme der Qualitätssicherung, die schon vor der Konferenz mit den für die Diagnose des Pathologen wichtigen klinischen Angaben (z. B. bisherige Therapie) beginnt. Überlegungen zu Anforderungen an Standards und Qualität werden im vorliegenden Heft kooperativ von einem Pathologen und Urologen gemeinsam dargestellt.

Gleichzeitig wollen wir mit dem vorliegenden Heft verdeutlichen, dass die Pathologie einem Wandel unterliegt, den die klinischen Partner kennen sollten und unterstützen können: Über die konventionelle Histomorphologie hinaus hilft die Immunhistochemie, und nun auch zunehmend die molekulare Diagnostik am Gewebe, den Tumor noch individueller zu charakterisieren und die Wirksamkeit von Medikamenten genauer als früher vor Einnahmebeginn zu definieren. Der Vision, dass der Pathologe in naher Zukunft in der Lage ist, ein dem Antibiogramm von bakteriellen Erregern angelehntes "Antionkogramm“ mit dem Befund zu ermitteln, kommen wir nun tatsächlich durch die Verfügbarkeit neuer und v. a. schneller Genom- und Ana- 
lysetechniken näher. Es erscheint zunehmend plausibel dass molekular-pathologische Untersuchungen das Ansprechen auf viele pharmazeutisch bereits entwickelten Target-Therapeutika gegen Zielstrukturen des individuellen Tumors vorhersagen können.

Durch diese Vermittlung solcher $\mathrm{Zu}$ satzinformationen wird der Pathologe noch stärker als bisher ein Lotse für seine klinischen Kollegen und seine Arbeit zunehmend im Hinblick auf Therapiebeginn auch durch solche Zusatzdiagnostik zeitkritisch. Wir versuchen mit den beiden letzten Beiträgen erstens den Stand der sinnvollen „etablierten“ Zusatzdiagnostik für die drei Organtumoren zu schildern und zweitens dem Leser einen Ausblick für die laufenden Entwicklungen im Bereich der molekularen Zusatzdiagnostik zu geben. Pathologen sind hier Autoren gemeinsam mit Molekularbiologen. Es werden nicht nur die technischen Entwicklungen geschildert! Die Notwendigkeit des hochwertigen Biobanking, welches recht umfangreich staatlich gefördert wird und der Sinn der intensiven Teilnahme an klinischen Studien unter Einbeziehung der Gewebeanalyse (Histologie und molekulare Profile) werden plausibel gemacht und der Bezug zur täglichen Betreuung unserer Patienten wird offensichtlich.

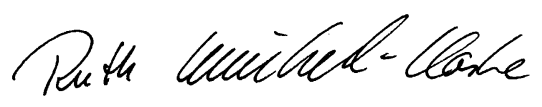

\section{R. Knüchel-Clarke}
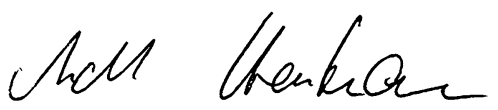

A. Hartmann

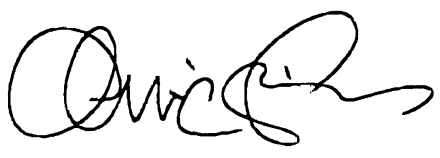

C. Stief

\section{Korrespondenzadresse}

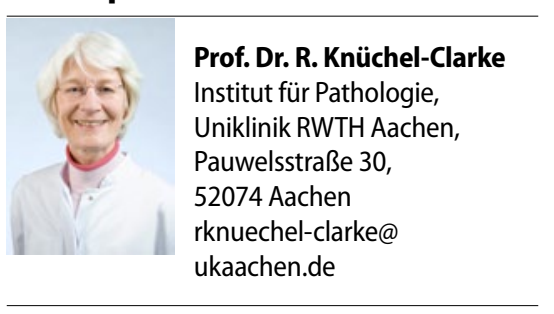

\section{GROßE FREIHEIT - liebe.lust.leben}

Neue Ausstellung zu HIV/AIDS und STI

Mit ihrer neuen Ausstellung "GROßE FREIHEIT - liebe.lust.leben." greift die Bundeszentrale für gesundheitliche Aufklärung (BZgA) ein Thema auf, das vielen Menschen überhaupt nicht oder noch viel zu wenig bekannt ist: Das Risiko, sich eine sexuell übertragbare Infektion zuzuziehen und zu erkranken.

HIV kennen die Menschen heute, aber die wenigsten wissen, was Syphilis, Chlamydien, Gonorrhoe, Hepatitis B, Hepatitis C, Herpes genitalis, Papillomavirus und Trichomonaden sind und dass sie zur Gruppe der STI gehören.

Deshalb stellt die BZgA diese Infektionen in der neuen, interaktiven Ausstellung in den Mittelpunkt und zeigt auf unkonventionelle Art, wie man sich vor sexuell übertragbaren Infektionen schützen kann. Für den markanten Ausstellungsraum wurden sechs Seecontainer zu umgebaut und durch ein aufblasbares Kuppelzelt ergänzt.

Die Besucher erhalten Einblick in das Leben von acht Menschen. Jeder der Charaktere erzählt eine individuelle Geschichte, in der er die Besucher mit dem Risiko, an sexuell übertragbaren Infektionen zu erkranken, konfrontiert.

Die BZgA hat die Ausstellung als ein zentrales Element ihrer erfolgreichen Schutzkampagne „mach's mit - Wissen \& Kondom" entwickelt. An den Einsatzorten wirken Fachstellen kooperativ bei den Ausstellungseinsätzen mit, indem sie ihre Beratungsangebote aktiv einbringen. Mit der Ausstellung geht die BZgA ganzjährig bundesweit auf Tour. In diesem Jahr macht sie neben Berlin in acht weiteren deutschen Großstädten Station.

Quelle: Bundeszentrale für gesundheitliche Aufklärung, www.bzga.de 